Rev. Elev. Méd. vét. Pays trop., 1976, 29 (3) : 267-275.

\title{
Les feux courants et l'élevage en savane soudanienne
}

\author{
par P. GRANIER (*) et Y. CABANIS
}

\begin{abstract}
RÉSUMÉ
Les auteurs ont étudié les rapports existant entre les feux courants et le mode d'élevage extensif. Une expérimentation sur les effets du feu en savane et sur les conséquences de sa suppression montre que ce facteur écologique est responsable du maintien de la productivité de la strate herbacée. Sur le plan nutritionnel, 1 l est indispensable pour assurer au bétail un apport en matières azotées en fin de saison sèche et améliorer l'appétibilité de l'herbe.

A partir de l'étude de la bıologie des espèces de savane, une technique d'exploitation intégrant les aspects positifs du feu et réduisant ses effets érosifs a été mise au point ; elle est basée sur l'emploi dans une rotation du feu à contresaison.
\end{abstract}

\section{INTRODUCTION}

Les régions soudaniennes, caractérisées par une saison sèche longue de 7 à 8 mois, et une pluviosité comprise entre 1000 et $1500 \mathrm{~mm}$, portent des savanes où le peuplement graminéen est essentiellement formé d'espèces cespiteuses pérennes. L'élevage des bovidés y est couramment pratiqué selon le mode extensif.

On constate que, partout, les éleveurs mettent à feu périodiquement ces savanes. Lorsque la végétation avant les pluies est très desséchée, ces feux courants, communément dénommés « feux de brousse » peuvent prendre une très grande extension. Ils échappent fréquemment à leurs auteurs et peuvent ravager des cultures, des forêts, des villages même. Aussi les considère-t-on généralement comme une calamité. Des lois souvent draconiennes les ont partout interdits. Mais nulle part elles ne sont parvenues à les supprimer, voire à les atténuer.

Si l'action pro-érosive des feux sur les sols n'est pas plus niable que les autres méfaits, il

(*) Adresse actuelle: Laboratoire de l'Elevage B. P. 485 Niamey, République du Niger. faut se demander quelles sont les raisons de leur persistance, et tenter de juger plus justement ce qu'it faut porter à leur débit et à leur crédit.

C'est pourquoi nous avons entrepris des expérimentations ayant pour but de préciser les actions du feu sur les sols, la végétation, et sur l'équilibre phytosociologique des savanes soudaniennes.

Les résultats expérimentaux seront capables, nous le verrons, de nous diriger vers une exploitation améliorée des pâturages, conciliant une charge optimale en bétail et le maintien de l'équilibre sol-plante-animal. Dans les conditions d'élevage extensif, seule une utilisation rationnalisée des feux permet d'y parvenir.

\section{I. ÉTUDE EXPÉRIMENTALE DE L'ACTION DU FEU}

Le passage du feu dans une savane a une action directe et immédiate sur la végétation, une action directe sur les sols et des effets éloignés sur la modification de l'équilibre sols/végétation. 
Des études ont été effectuées au Centre de Recherches Zootechniques et Fourragères de Kianjasoa afin d'apporter une contribution à la connaissance des facteurs en cause.

\section{A. MÉTHODOLOGIE}

\section{Sur le terrain}

On a comparé l'évolution de la végétation (fréquence, recouvrement, germinations) sur des parcelles homogènes soumises ou non aux feux.

Une mise en défens absolue, pendant dix ans a permis de contrôler l'évolution des sols et de Ja végétation (études des profils, analyses chimiques, relevés en lignes).

Les effets sur le ruissellement ont été étudiés par BAILLY et collab. du C. T. F. T. qui ont installé des parcelles élémentaires représentatives de l'Effet des facteurs étudiés (feu, mise en défens).

Le problème important de la valeur alimentaire comparée des pâturages brûlés ou non en saison sèche a été abordé en comparant la croissance de troupeaux alimentés sur l'un ou l'autre.

\section{Au laboratoire}

L'analyse des effets des différents facteurs écologiques a été effectuée en soumettant les semences des espèces étudiées sur le terrain à l'action de la chaleur, du soleil, de l'humidité et $\mathrm{du}$ feu à différents niveaux. Des tests de germinations et l'étude du mécanisme de la germination ont permis de préciser le rôle du feu sur les arêtes et les glumes dis diaspores. Ces travaux ont fait l'objet d'une publication particulière (9).

\section{B. ACTION SUR LA VÉGÉTATION}

\section{Effets immédiats}

\section{a) Levée de dormance}

Le feu en brûlant l'appareil aérien entraîne une élévation de température de l'ordre de 60 à $80^{\circ}$ au niveau du sol. Tout est détruit audessus de $5 \mathrm{~cm}$ de hauteur; seuls résistent les bourgeons ou les graines possédant des enveloppes protectrices. Ces deux modes de résistance sont précisément utilisés par les graminées de savane.

Action sur les bourgeons : chez les pérennes hémicryptophytes, les bourgeons basilaires sont entourés par la base dis gaines et placés au milieu des chaumes développés. Ce sont autant de protections que le feu supprime et qui mettent les bourgeons à nu.

Ensuite la disparition de l'appareil végétatif et des bourgeons supérieurs supprime la dominance de ces derniers et lève acc:dentellement l'inhibition.

La comparaison de deux parcelles, l'une brûlée tous les ans, l'autre fauchée au même moment montre un démarrage plus rapide de la première parce que la levée d'inhibition est plus complète (8).

Action sur les graines : la plupart des esfèces savanicoles produisent et disséminent leurs graines avant le passaga du feu. Comme pour les bourgeons, celui-ci détruit tous les appendices de la diaspore et amenuise les protections tégumentaires.

Les obstacles à la pénétration de l'eau étant levés, la germination s'en trouve donc favorisée.

\section{b) La printanisation et l'action sur la croissance}

$\mathrm{Si}$ la germination des graines dépend uniquement de la présence de l'eau dans le milieu, et ne peut se produire qu'au moment des premières pluies, la reprise d'activité de la touffe de graminées est indépendante des conditions climatiques et peut ainsi se manifester à n'importe quelle période de l'année.

C'est donc que nous avons affaire à une répétition des conditions normales du réveil saisonnier des végétaux. L'éclosion des bourgeons et la reprise de l'activité phytosynthétique de la plante sont une conséquence de l'apport de chaleur brutal par le passage du feu. Celui-ci a fourni en un temps très court l'équivalent des sommes de chaleur nécessaires au démarrage printanier des bourgeons.

Ce choc thermique entraine la mobilisation des substances de réserves (nitrates) accumulées dans les racines et de l'eau disponible dans les tissus de la plante. La croissance des talles innovées est immédiate.

La printanisation par le feu est un réveil systématique des plantes adaptées. Elle entraîne une croissance limitée dans l'espace et le temps selon les ressources disponibles dans la plante et les conditions de milieu.

c) Action sur le port et le type biologique

Les formations végétales entretenues par le 
feu comprennent des types biologiques sélectionnés.

Parmi les herbacées, on y retrouve des annuelles (thérophytes), des hémicryptophytes et des géophytes. Les bourgeons enterrés ou enveloppés sont résistants au feu. Les graines supportent bien l'élévation de température accidentelle.

Le développement de la plante s'effectue entre deux feux. L'appareil aérien est détruit régulièrement.

L'intervention fréquente des brûlis a donc favorisé le développement des plantes les plus résistantes parmi toutes les formes biologiques présentes. Les graminées, et parmi elles les Andropogonées, ont fourni de nombreux types d'adaptation. C'est pourquoi cette famille est abondamment représentée dans les peuplements savanicoles.

\section{d) Action sur les germinations}

Dans une autre étude nous avons conclu que la capacité de dissémination des graminées de savane est très élevée. Ces expèces produisent une grande quantité de semences dont le pouvoir germinatif est excellent. Leur capacité de germination est adaptée aux facteurs écologiques, ce qui explique que ces espèces ont une aire de dispersion très étendue et que certaines soient pantropicales.

Il n'y a pas d'inhibition qui ne soit levée au cours de la saison sèche qui suit leur formation. La maturation des graines par exposition au soleil et à la chaleur prolongée améliore le pourcentage de levées.

La protection tégumentaire est d'autant plus forte que l'espèce possède une grande extension. Les arêtes (Andropogonées) jouent un rôle déterminant dans la germination. Pendant la saison sèche, elles favorisent la dispersion lorsque la graine tombe sur le sol, elles permettent, une fois le callus calé sur le support, à celle-ci de pénétrer. Les variations de l'humidité de l'air mobilisent la colonne qui imprime à la graine un mouvement de rotation. Il semble que pendant cette période, l'arête inhibe la germination en empêchant la pénétration de l'eau jusqu'à l'embryon. Ensuite, lorsque la graine s'est enfoncée, l'arête est détruite par le feu ou arrachée par le piétinement du bétail. La pénétration de l'eau déclenche jmmédiatement la germination, mais la vitesse de pénétration est plus ou moins rapide selon les espèces et ce caractère permet de distinguer parmi ces graminées que l'on rencontre dans une même formation herbacée, des adaptations plus prononcées au milieu humide, ce qui autorise à leur attribuer une origine forestière (Aristida, Imperata).

La régénération des pâturages ne dépend pas essentiellement des capacités de germinations des espèces fourragères mais plutôt de l' " ouverture » du milieu. Le contrôle des levées en savane montre que le nombre de plantules est lié à la pression des facteurs biotiques dont les principaux sont le feu et le pâturage. Pour que la structure de l'association végétale se maintienne en équilibre, il est nécessaire que celle-ci soit parcourue régulièrement par les feux et exploitée par le bétail. La mise en défens expérimentale conduit à la disparition des espèces savanicoles.

Le feu agit par la minéralisation de la matière organique, la suppression de l'écran constitué par les litières et en favorisant la germination. Le bétail fixe les graines au sol et favorise le tallage.

La mise en défens prolongée d'une savane aboutit à une modification de sa composition floristique qui se traduit par une diminution de ses potentialités sur le plan fourrager, puisque la compétition fait disparaître les espèces savanicoles qui sont les espèces fourragères au profit des espèces du milieu forestier.

\section{Effets éloignés}

Un équilibre fluctuant est sans cesse remis en question par la pression des facteurs. C'est ainsi qu'on assiste à l'appauvrissement en espèces de la formation et à une augmentation de la fréquence des types les mieux adaptés. L'Heteropogon contortus étend son aire et sa dominance grâce à des mécanismes divers. L'Aristida rufescens peut constituer des peuplements monospécifiques dans des stations très appauvries où la formation de savane n'a pu se maintenir.

Le feu modifie le recouvrement total à chaque passage, en supprimant les ligneux suffrutescents dont l'introduction modifie le peuplement et amène un changement $\mathrm{du}$ stade végétal (évolution progressive).

Il remet en cause régulièrement la compétition dans le milieu en favorisant les espèces à croissance et recouvrement rapides: les herbacées aux dépens des ligneux, et parmi les graminées 
Statıon de Kianjasca

\begin{tabular}{|c|c|c|c|c|c|c|c|c|c|c|c|}
\hline \multirow{3}{*}{ Carpagnes } & \multirow{3}{*}{ Pluies } & \multirow{3}{*}{$\begin{array}{l}\text { Ip } \\
\text { (êrosivité) } \\
\text { (unités LSA) }\end{array}$} & \multicolumn{9}{|c|}{$P A R C E L L E S$} \\
\hline & & & \multicolumn{2}{|c|}{$\frac{2}{\text { Sutyiosantines }}$} & \multicolumn{2}{|c|}{$\begin{array}{c}3 \\
\text { Pâturage nature1 } \\
\text { fauché }\end{array}$} & \multicolumn{2}{|c|}{$\begin{array}{c}4 \\
\text { Pâturage naturel } \\
\text { brûlê (3 fois) }\end{array}$} & \multicolumn{2}{|c|}{$\begin{array}{l}5 \\
\text { Pâturage naturel } \\
\text { brûilê (1 fois) }\end{array}$} & \multirow{2}{*}{$\begin{array}{c}\begin{array}{c}\text { W } \\
\text { Jachère } \\
\text { travaillée }\end{array} \\
\begin{array}{c}\text { P.T. } \\
\text { t/ha }\end{array}\end{array}$} \\
\hline & & & $\begin{array}{l}\mathrm{R} \\
\mathrm{m} \\
\text { hil }\end{array}$ & $\begin{array}{l}\text { P.T. } \\
\text { tha }\end{array}$ & $\begin{array}{l}R \\
\mathrm{mmm}\end{array}$ & $\begin{array}{l}\text { P.T. } \\
\text { t/ha }\end{array}$ & $\begin{array}{l}\mathrm{R} \\
\mathrm{nm}\end{array}$ & $\begin{array}{l}\text { P.T. } \\
\text { t/ha }\end{array}$ & $\begin{array}{l}\mathrm{R} \\
\mathrm{m}\end{array}$ & $\begin{array}{l}\text { P.T. } \\
\text { t/ha }\end{array}$ & \\
\hline $1967 / 68$ & 1574,5 & 514,99 & 34,05 & 3,390 & 66,48 & 0,052 & 139,12 & 0,902 & 179,72 & 2,406 & 84,133 \\
\hline $1968 / 69$ & 1825,4 & 644,24 & 5,45 & 0,126 & 51,31 & 0 & 70,82 & 0 & 98,69 & 0 & 326,538 \\
\hline $1969 / 70$ & 1453,1 & 560,43 & 3,23 & 0,110 & 62,23 & 0 & 122,87 & 0,336 & 114,48 & 0 & 195,751 \\
\hline $1970 / 71$ & $1675, B$ & 956,00 & 41,42 & 0 & 171,34 & 0 & 260,24 & 1,203 & 204,93 & 0 & 296,488 \\
\hline $\begin{array}{l}\text { Total } \\
4 \\
\text { campagnes }\end{array}$ & 6528,8 & 2675,66 & 84,15 & 3,626 & 351,36 & 0,052 & 593,05 & 2,441 & 597,82 & 2,406 & 902,910 \\
\hline
\end{tabular}

$\mathrm{R}=$ russellement ; P.T. = pertes en terre (en tonnes par hectare).

celles qui sont les plus agressives, soit les espèces à grande extension: Heteropogon contortus, Hyparrhenia rufa, Chrysopogon serrulatus.

L'étude des mécanismes d'adaptation montre que le feu en supprimant les litières et en minéralisant l'humus provoque un dessèchement du milieu, et que les espèces qu'il sélectionne sont essentiellement des xérophytes, le terme de pyrophytes étant semble-t-il trop restrictif (15).

\section{ACTION SUR LES SOLS}

L'expérimentation concernant l'action des feux sur les sols a été effectuée, à notre demande, par la Division Sol-Forêt du Centre technique forestier tropical sur des parcelles élémentaires implantées sur les pâturages qui font l'objet de la présente étude (3). Les résultats sont réunis sur le tableau ci-après.

Il faut noter que l'action du feu a été étudiée sur deux parcelles de pente voisine de 7 p. 100 :

- l'une a été brûlée pendant trois années consécutives en fin de saison sèche;

- l'autre a été brûlée à la mise en place du dispositif (1967) puis maintenue en défens.

Il apparaît que l'érosion, caractérisée par les pertes en terre, est intense après le passage du feu. Mais la stabilisation est rapide. "Les courbes cumulées des pertes en fonction de l'index de pluie (= érosivité) présentent toutes une recrudescence de l'érosion après le brûlis puis une atténuation progressive au fur et à mesure que le couvert se referme. "

« La reconstitution du couvert après le feu suffit à supprimer totalement l'érosion et à augmenter de façon sensible l'infiltration. ”

A partir de ces constatations, nous nous sommes efforcés de mettre au point une technique qui permette d'intégrer le feu en tant que facteur écologique indispensable pour les raisons qui ont été développées dans le chapitre précédent, mais de limiter les effets érosifs sur les sols. Puisque la reconstitution du couvert suffit à supprimer l'érosion, la mise à feu à contre-saison doit permettre de supprimer les refus lignifiés et d'éviter les départs de terre, parce que :

- on brûle une strate sèche, mais le sol reste protégé par une strate verte ; feu ;

- les pluies sont peu importantes après le - le couvert s'est reconstitué lorsque šinstalle la saison des pluies suivante.

\section{II. ÉTUDE EXPÉRIMENTALE DES EFFETS DE LA SUPPRESSION DES FEUX}

Elle peut être observée sur des pâturages fauchés tous les ans et sur des parcelles mises en défens.

\section{Effets sur la végétation}

Lorsque la biomasse est enlevée régulièrement dans le cas du fauchage annuel, on entretient et on stimule les types hémicryptophytes. La compétition tourne à l'avantage de l'espèce ayant un recouvrement basal important ou 
possédant un tallage actif. Les aires de fauche montrent une dominance d'Heteropogon et d'Hyparrhenia. Le pâturage du bétail conduit aux mêmes effets quand il n'est pas excessif. La formation savanicole se maintient grâce à l'équilibre des facteurs en place. Lorsque la biomasse n'est pas supprimée dans le cas d'une mise en défens, il y a une accumulation des litières et augmentation du recouvrement total. La modification du couvert n'est pas favorable aux espèces cespiteuses car la compétition n'est plus une question d'occupation du sol mais de l'espace aérien. Les types biologiques compétitifs vont surcimer les espèces inférieures et occuper des strates de plus en plus hautes. Comme parmi les vivaces les graminées ne comprennent pas de types biologiques adaptés, on assiste au développement de ligneux suffrutescents parmi lesquels on trouve beaucoup de légumineuses.

La modification du peuplement est caractérisée par un changement de la stratification, un remplacement des types biologiques et un renouvellement des espèces.

Le réembroussaillement des savanes est un processus bien connu. Une parcelle de pâturage naturel à Hyparrhenia et Heteropogon dominant mise en défens pendant dix ans a vu le remplacement des deux graminées de savanes par des herbacées géophytes et par des ligneux pérennes. L'évolution rapide est due à des conditions édaphiques favorables. Elle est plus lente dans les zones dégradées mais plus avancée dans les stations protégées ou inaccessibles à l'homme (talwegs, colluvions). L'évolution vers une formation forestière dépend, à ce stade, de la présence de porte-graines et des capacités de dissémination et de germination de celles-ci.

\section{Effets sur les sols}

L'accumulation de litière supprime les effets du ruissellement et empêche le dessèchement rapide de l'horizon superficiel.

L'humidification entraîne une reconstitution et un épaississement de l'horizon A. Un enrichissement en matière organique est constaté. La conséquence la plus intéressante est l'amélioration de l'ensemble du profil, et une meilleure porosité. Les racines prospectent de plus en plus profondément et finissent par pénétrer la couche compacte. Cet accroissement de la biomasse des racines a une influence directe sur l'enrichissement du sol. Le phosphore des racines donne des composés phospho-humiques et d'après
HEDIN (11) 80 p. 100 de l'azote des racines est minéralisé. On retourne ainsi à des sols profonds qui ont des caractéristiques et une vocation forestières. Ainsi la mise en défens provoque une mutation de la vocation de la zone dans laquelle le pâturage extensif doit être abandonné. Le couvert végétal et les sols sont tellement modifiés, et en particulier les sols dans le sens de l'enrichissement que cela implique une modification de l'exploitation et l'adoption d'un mode d'intervention intensif.

\section{LES FEUX TRADITIONNELS DE SAISON SĖCHE}

Les résultats des expérimentations citées plus haut nous permettent de comprendre le comportement de l'éleveur qui a une connaissance empirique des faits. Selon les cas, le motif de la mise à feu est l'un ou plusieurs des arguments suivants :

\section{Lutte contre le réembroussaillement}

C'est-à-dire l'élimination des buissons et arbustes qui se développent dans un pâturage laissé en défens pendant plusieurs années (4 à 5 ans suffisent).

Dès qu'un déséquilibre est amorcé, n'importe quelle espèce adaptée aux nouvelles conditions peut se propager si elle produit des diaspores ayant un bon pouvoir germinatif en quantité suffisante.

\section{Enlèvement des litières}

Il correspond à la fauche des refus dans l'exploitation des pâturages artificiels. Les litières. produites par les refus des cycles végétatifs précédents, versent, pourrissent et gênent la préhension des jeunes pousses. Dans certaines savanes à productivité élevée, cette masse végétale en décomposition dégage des odeurs qui rebutent le bétail.

\section{Printanisation}

Nous avons déjà montré qu'un pâturage brûlé reverdissait et que la croissance de l'herbe sur ces zones était stimulée, ce qui, en quelque sorte, est un moyen d'avancer la saison des pluies.

\section{Amélioration de la valeur nutritive}

Si l'éleveur ne dispose pas de pâturages sur 
terres alluvionnées, ou de bas-fonds constamment humides, la ration du bétail en fin de saison sèche ne contient ni carotènes (provitamine A), ni matières azotées. Seuls les regains précoces peuvent fournir ces éléments indispensables qui n'existent que dans l'herbe jeune. Certains éleveurs affirment que sur des pâturages non brûlés, les vaches n'ont pas assez de lait pour alimenter les veaux et il est possible que nous retrouvions dans cette argumentation l'influence d'une carence azotée.

Au cours d'une expérimentation sur les divers modes de supplémentation des bovins en saison sèche, faite au C. R. Z. F. de Kianjasoa, on a observé, sur un lot témoin de bøufs adultes ne disposant que d'un pâturage naturel non brûlé depuis quatre ans, un amaigrissement moyen de $66,5 \mathrm{~kg}$, les extrêmes ayant atteint des pertes de poids de $87 \mathrm{~kg}$ avec 40 p. 100 de mortalités dues à la dénutrition.

Le contrôle de la croissance des zébus, au C. R. Z.F. de Miadana en saison des pluies met en évidence l'influence du feu sur les gains de poids. Alors que des zébus d'un an grossissent en moyenne de $43 \mathrm{~kg}$ pendant la saison des pluies, des lots maintenus sur des pâturages non brûlés ont accusé un gain moyen de $8 \mathrm{~kg}$ seulement.

\section{L'équilibre feux-végétation-bétail}

Il semble que l'on puisse considérer le feu comme cause favorisante dans l'évolution de l'équilibre de l'écosystème. La cause déterminante serait, à notre avis, le surpâturage des regains avant l'installation des pluies. Des zones d'élevage se maintiennent en équilibre malgré le passage répété des feux courants, et l'observation de la végétation et des troupeaux à différentes époques de l'année nous a permis d'avancer l'hypothèse suivante :

Une partie seulement des pâturages brûle en saison sèche et se recouvre d'une herbe verte précocement. Le bétail, qui a besoin de matières azotées et de carotènes, et qui préfère pâturer de l'herbe rase, se concentre sur ces regains, délaissant les zones non brûlées, lignifiées. Cette concentration provoque un surpâturage et les coupes répétées par la dent du bétail finissent par épuiser les touffes qui, en l'absence des pluies, ne produisent qu'à partir des réserves accumulées dans les racines au début de l'hiver (ou saison sèche). Lorsque la saison des pluies s'installe, ces touffes épuisées ne peuvent pas faire un cycle végétatif normal, mais donnent une strate basse sur laquelle'le bétail continue à pâturer. Lorsque les pluies s'arrêtent, ces zones recouvertes d'une végétation rasée (qui fleurit en donnant parfois des inflorescences aberrantes) ne laisseront pas passer les feux courants en saison sèche, les matières combustibles étant en quantité insuffisante. Elles se mettront naturellement en défens et ce sont les parties non brûlées les années précédentes, qui donc ont été délaissées par le bétail et qui sont recouvertes de pailles sèches, qui vont brûler et le cycle recommencera. Ceci permet d'expliquer pourquoi les feux s'arrêtent naturellement selon une ligne sans causes apparentes dans des savanes homogènes, et pourquoi l'équilibre entre la végétation et le bétail peut se maintenir, la mise en défens par rotation naturelle permettant une reconstitution des réserves dans le sol et les racines des graminées. La mise à feu des pâturages existe depuis toujours, mais les surfaces brûlées étant de beaucoup supérieures aux besoins, le bétail ne peut surpâturer la totalité des regains et le potentiel herbacé se reconstitue.

\section{Le déséquilibre feux-végétation-bétail}

Dans certaines régions, la pression de certains facteurs écologiques peut provoquer une évolution de cet équilibre, les causes sont les suivantes :

a) Causes favorisantes

- l'accélération du peuplement due à la démographie et à l'immigration vers les terres cultivables ;

- Ia mise en cultures des terres riches;

- la réduction des surfaces pâturables;

- la disparition des pâturages de saison sèche, conséquence directe de la mise en culture des terres riches;

- la sédentarisation de l'élevage rendue nécessaire à cause de la dispersion des zones cultivées ;

- l'élévation de la charge à l'hectare sur les zones où le bétail est refoulé, et donc l'accroissement du « surpâturage » sur la végétation, en particulier sur les regains.

Si la quantité de matières combustibles est insuffisante, le feu est incapable de tuer les jeunes plants qui ont tendance à se disperser dans la savane.

b) Causes déterminantes

Parvenue à ce stade, l'évolution dépend de 
l'existence de porte-graines dans la savane et des caractéristiques biologiques de ces espèces. La production de graines, leur facilité de dispersion, leur pouvoir germinatif élevé, leur besoin de lumière sont autant de facteurs qui augmentent les risques d'invasion dans un milieu où le feu a réduit la compétition interspécifique. De plus, le défrichement par l'homme joue, même s'il ne concerne que de petites surfaces, le rôle de catalyseur parce que les jachères constituent un milieu propice et que le défrichement respecte les arbres les plus gros qui sont les principaux producteurs de semences.

Ce processus aboutit à la reconstitution de formations ligneuses secondaires reconnaissables au fait qu'elles sont monospécifiques et que les arbres ont le même âge dans une zone donnée. Les principales familles sont celles dont les semences sont dispersées par le vent (Bignonacées, Composées) ou par le bétail (Légumineuses).

\section{LES FEUX DE CONTRE-SAISON}

Devant la nécessité d'utiliser le feu, on a cherché à mettre au point des techniques qui, tout en éliminant les refus et contrôlant le réembroussaillement, permettraient de limiter la dégradation des sols. On a défini ce que l'on a appelé les «feux à contre-saison ». Mais si cette technique répondait aux exigences que l'on vient de citer, par contre sur le plan nutritionnel, elle n'apportait aucune amélioration parce que les dates de mises à feu étaient calculées de telle façon que la hauteur des pluies qui tombaient après la mise à feu était suffisante pour permettre l'évolution d'un cycle végétatif normal. L'étude expérimentale du feu et de la biologie des espèces nous a permis de définir une date de mise à feu (10) : elle doit se situer avant la fin des pluies pour permettre la croissance des regains mais elle doit être relativement tardive pour que l'insuffisance d'eau provoque la formation de cycles limités en hauteur, donc pauvres en tissus de soutien lignifiés indigestibles. Ce blocage de la végétation à un certain stade assure la persistance de l'appétibilité et d'un certain taux de digestibilité. Cette technique est basée sur l'utilisation de la rotation et du feu à contre-saison.

La rotation permet à la fois de contrôler l'évolution de la végétation et de réduire les écarts saisonniers sur le plan de sa valeur nutri- tionnelle. En saison chaude et pluvieuse, la productivité de l'herbe est très élevée et toujours supérieure aux capacités d'ingestion du bétail, ce qui amène la production de refus. On peut donc dire que si la quantité d'herbe consommable est supérieure aux besoins il n'est pas nécessaire et souhaitable, en saison des pluies, d'attribuer au bétail la totalité des surfaces disponibles. Il est préférable de concentrer le cheptel sur certaines parcelles et laisser inexploitée une certaine superficie qui, brûlée à contresaison, sera pâturée en saison sèche. Le pâturage est divisé en trois zones qui correspondent aux trois périodes suivantes :
$A$ : début des pluies $\rightarrow$ montaison
B : montaison $\rightarrow$ début de lignification et dispersion des semences
$\mathrm{C}$ : saison sèche.

Le bétail effectue une rotation sur les trois parcelles pendant les époques indiquées cidessus.

La mise à feu est effectuée lorsqu'il est tombé environ les $3 / 4$ de la pluviométrie annuelle, et le pâturage laissé en défens jusqu'au début de la saison sèche. On brûle la parcelle qui a été " débrayée " au cours de la saison des pluies précédente et sur laquelle la végétation a eu la possibilité de terminer son évolution. Chaque parcelle passe par une phase de repos permettant la reconstitution des réserves racinaires, la minéralisation de la matière organique et le réensemencement naturel.

Cette technique permet de maintenir des parefeux économiques, les parcelles brûlées à contresaison et pâturées en saison sèche ne pouvant pas rebrûler dans l'année. Les charges instantanées, puisqu'elles sont trois fois plus fortes que dans l'élevage traditionnel réalisent une homogénéisation de la strate herbacée, traitement obtenu par emploi du rotary-cutter en élevage intensif.

La périodicité des feux, le respect des temps de repos et la mise à feu à la fin des pluies permettent d'éviter la dégradation des sols, la carence en azote et le déséquilibre de la végétation. Un contrôle de l'efficacité de cette technique sur le plan de la production de viande et du maintien de la structure de l'association végétale a été effectué pendant un cycle de quatre années et a donné lieu à une mise au point publiée par ailleurs (10). 


\section{CONCLUSION}

Dans une savane de type soudanien, soumise à une saison sèche, on remarque qu'à la fin des pluies, lorsque les cycles végétatifs s'arrêtent à la fructification, les cycles suivants sont déjà représentés par les premières feuilles basilaires. Mais le manque d'eau et l'inhıbition maintenue par les chaumes persistants font que ces cycles ne se développent pas avant que les pluies s'installent. Le passage du feu permet le démarrage de la végétation indépendamment de l'époque en levant les inhibitions. Les éleveurs ont donc à leur portée un moyen économique pour faire pousser l'herbe avant la saison des pluies. C'est une des causes essentielles des mises à feu, avec l'élimination des refus et la lutte contre le réembroussaillement.

Pendant longtemps, on n'a voulu voir dans le feu que son influence dégradante sur les sols et les formations forestières. Mais il ne faut pas perdre de vue, qu'en savane, c'est un facteur écologique normal, puisqu'il est responsable du maintien de la strate herbacée, de la régénération des espèces typiquement savanicoles que sont les espèces fourragères.

La suppression du feu dans ce milieu provoque la mutation de la formation herbacée vers une formation de transition dans le sens du retour au climax, et donc la suppression de l'élevage.

Il n'est donc pas possible de préconiser l'interdiction du feu dans les zones où l'élevage ne peut se maintenir que sous une forme extensive.

Il nous a paru plus logique, plutôt que de nier toute valeur au mode d'exploitation empirique, d'étudier objectivement les motifs des éleveurs, et à partir de la connaissance des faits de mettre au point une technique qui intègre les aspects positifs et réduit au maximum les effets érosifs.

Cette technique utilise le feu à contre-saison dans une exploitation basée sur la rotation.

Un contrôle rigoureux de l'évolution de la végétation a montré que le feu améliorait les capacités de germination des graminées de savane et que la dégradation des sols était temporaire, l'érosion s'atténuant dès que le couvert se refermait.

Il est certain que si les conditions socioéconomiques évoluent, l'adoption d'un mode d'exploitation plus intensif est préférable, et la suppression des feux dans ce contexte modifie la vocation de la zone considérée.

\section{SUMMARY}

\section{Fires and animal husbandry in sudanese savannah}

The authors have studied the relations between fires and extensive anımal husbandry conditions. An experimentation on fire effects in savannah and the results of its suppression shows that this ecological factor is responsible for the keeping of herbaceous stratum productivity. From the point of view of nutrition, the fire is necessary to ensure the livestock to have nitrogen feed at the end of dry season and to improve the grass palatability.

From the study of savannah species biology, an utilization method including the positive aspects of fire and reducing its erosive effects was perfected; its base is the uttlization of a fire rotation out of season.

\section{RESUMEN}

\section{Los fuegos y la ganaderia en sabana sudanesa}

Los autores estudiaron las relaciones existiendo entre los fuegos y el modo de ganaderia extensiva.

Una experimentación sobre los efectos del fuego en sabana y sobre las consecuencias de su supresión muestra que este factor ecologico es responsable del mantenimiento de la productividad del estrato herbáceo. Desde el punto de vista nutricional, el fuego es indispensable para asegurar al ganado una aportación de materias nitrogenadas al fin de la estación seca y mejorar la apetecibilidad de la hierba.

A partir del estudio de la biologia de las especies de sabana, se ha mejorado una técnica de explotación integrando los aspectos positivos del fuego y reduciendo sus efectos erosivos ; y cuya base es el empleo del fuego fuera de estación en un pastoreo. 


\section{BIBLIOGRAPHIE}

1. ADJANOHOUN (E.). Etude phytosociologique des savanes de basse Côte-d'Ivoire. Vegetatio, 1962, 11: $1-38$.

2. ADJANOHOUN (E.). Végétation des savanes et des rochers découverts en Côte-d'Ivorre centrale. Paris, O. R. S. T. O. M., 1964. (Mémoire 7) 178 p. 32 pl. -39 p.

3. CENTRE TECHNIQUE FORESTIER TROPICAL (C. T. F. T.). Division Sol-Forêt. Résultats des parcelles élémentaires de la Statıon de Kianjasoa. Rapport. Juin 1972.

4. DESCOINGS (B.). Méthode de description des formations herbeuses intertropicales par la structure de la végétation. Candollea, 1971, 26 (2) : 223-257.

5. DEZ (J.). Les feux de brousse (Madagascar). Limitation, régression, démographie, mise en cultures. Bull. Madagascar, 1970 (295) : 926.

6. DOMMERGUES (Y.). Influence du défrichement de forêt suivi d'incendie sur l'activité biologique du sol. Mém. Inst. Sci. Madagascar, sér. D, 1952, 4 (2) : 273-293.

7. GRANIER (P.). Le rôle écologique de l'élevage dans la dynamique des savanes à Madagascar. D. E. S. Université de Tananarive, avril 1967.

8. GRANIER (P.), LAHORE (J.) et DUBOIS (P.) Etude du pâturage naturel à Madagascar. Productivité, conséquences pratiques. Rev. Elev. Méd. vét. Pays trop., 1968, 21 (2): 203-217.

9. HEDIN (L.). Influence des racines sur la teneur en matière organique du sol. Fourrages, 1972 (50) : 83-96.

10. HENRARD. Réactions de la microflore du sol aux feux de brousse. Bull. I. N.E. A. C., 1939 (20).

11. HUMBERT (H.). La destruction d'une flore insulaire par le feu. Principaux aspects de la végétation à Madagascar. Mém. Acad. Malg. 1927, 5, 79 p.

12. HUMBERT (H.). Les aspects biologiques du problème des feux de brousse et la protection de la nature dans les zones intertropicales. Inst. Roy. Colon. Belg. Bull. Sci., 1939, 9 (3): 811-835.

13. LEBRUN (J.). La végétation de la plaine alluviale au Sud du Lac Edouard. Inst. des Parcs Nationaux, Congo belge, Bruxelles, 1947, 800 p.

14. LEBRUN (J.). Les formes biologıques dans la végétation tropicale. Coll. Morph. Montpellier, 1965.

15. LOUW (J. G.). The influence of frequency of cuttıng on the yield chemical composition, digestibility and nutritive value of some grass species. Onderstepoort J. vet. Sci., 1938, $11: 163-244$.
16. MASSON (H.). La température du sol au cours d'un feu de brousse au Sénégal. Agron. trop., 1948, 3 (3-4) : 174-179.

17. McMECKAN (C. P.), Grazing management. Proc. N. Z. Soc, anim. Prod. $21: 47-60$.

18. McWILLIAM (J. R.), SHANKER (K.) et KNOX (R. B.). Effects of temperature and photoperiod on growth and reproductive development in Hyparrhenia hirta. Aust. J. agric. Res., 1970, 21 : 557-569.

19. MONNIER (Y.). Les eflets des feux de brousse sur une savane préforestière de Côte-d'Ivoire. Abıdjan, Dir. Rech. scient., 1968. 260 p. (Etud. eburn. IX).

20. MORAT $(\mathrm{Ph}$.$) . Contribution à l'étude des savanes$ du Sud-Ouest de Madagascar. Thèse Doct. es Sci. Nat. Faculté Paris-Sud (Orsay), $\mathrm{n}^{\circ}$ 985, série A, Juin 1972.

21. PEYRE DE FABREGUES (B.) et ROSSETTI (Ch.). Evolution des pâturages naturels sahéliens du Sud Tamesna. Maisons-Alfort, IEMVT, 1971, $135 \mathrm{p}$. (Etude agrostologique $n^{\circ} 32$ ).

22. PITOT (A.). Feux sauvages, végétation et sols en A. O. F. Bull. Inst. fr. Afr. noire, 1953, 15 (4) : 1369 1383.

23. PITOT (A.) et MASSON (H.). Quelques données sur la température au cours des feux de brousse aux environs de Dakar. Bull. Inst. Fr. Afr. noire, 1951, 13 (3) : 711-732.

24 ROBYNS (W.). Considérations sur les aspects biologiques des feux de brousse au Congo belge et au Ruanda Urundi. Bull. Inst. Roy. Col. Belge, 1938, $9: 383-420$.

25. SCHNELL (R.). Introduction à la phytogéographie des pays tropicaux. 2 vol. Parıs, Gauther-Villars, 1970, 1971. 499 p., 952 p.

26. SILLANS (R.). Les savanes de l'Afrique Centrale Française. Encycl. Biol., Paris, 1958, 423 p.

27. SMITH (C. A.). Studies on the Hyparrhenia veld of Zambia. 7. The effects of cattle grazing veld and dambo at different stocking rates. J. agric. $\mathrm{Scl}$, Camb., 1966, 66 pr. 1, 49-56.

28. STURTZ (J.). Burning and pasture establishment in the Northern territory. Australia. Procecding 2 nd world conference on animal production Unıv. Maryland U. S. A., July 1968, 419-420.

29. VAN RENSBURG (H. J.). Fires and their effect on pastures. Addis-Abeba, F. A. O. 1964, (Working paper $n^{\circ} 5$ ).

30. VAN RENSBURG (H. J.). Grass burning experiments on the msima river stock farm, Southern Highlands, Tanganika. E. Afr. agric. J. 1952, 17 : 1-11. 